1015

\section{THE CRITICAL THINKING DISPOSITIONS AND EFFECTING FACTORS OF THE NURSES WORKING AT PEDIATRIC UNITS}

\author{
N. Cinar ${ }^{1}$, Ö. Dogu ${ }^{2}$, S. Kuguoglu ${ }^{3}$
}

${ }^{1}$ Sakarya University, School of Health Sciences,

2Sakarya Education and Research Hospital, Sakarya, Turkey, ${ }^{3}$ Acibadem University, Health

Science Faculty, Part Time Faculty, Sakarya, Turkey

Objective: This study is carried out in order to determine the critical thinking dispositions of the nurses working at the pediatric units and to determine the factors effecting these levels.

Method: The sample of this research is built up by the 36 nurses working for pediatric units in a State Hospital in Sakarya / Turkey (2010). Data were collected using a personel information form and California Critical Thinking Disposition Inventory ( CCTDI). In the analysis of the data mean, percentage, Mann Whitney $U$ Test and Kruskal wallis Test were used.

Results: It is determined that $38.9 \%$ of the respondent nurses was from neonatal intensive care unit, $36.1 \%$ was from general pediatric units, $47.2 \%$ was worked $1-5$ years in pediatric unit. The average critical thinking disposition score of the participants was found $253.03 \pm 19.86$. When the subscale scores were evaluated, the nurses were received the highest mean scores from the analyticity subscale $(48.33 \pm 4.99)$ and the lowest score from the self-confidence sub-scale (37.89 \pm 7.13).

Statistical no significant difference was determined among nurses' critical thinking disposition scores according to their working experiences, their working units $(p>0.05)$. However there was a statistical meaningful difference between the total score critical thinking dispositions of the nurses participate to service training activities $(p=0.019)$.

Conclusion: It was assigned critical thinking disposition scores of the nurses working at pediatric units are medium . However, participating in service training activities was determined to affect the critical thinking in a positive way.
1016

\section{TETRAHYDROBIOPTERIN RESPONSIVENESS AFTER LOADING TEST OF 21 CZECH HPA/PKU PATIENTS AND CORRELATIONS TO THEIR GENOTYPE}

D. Prochazkova ${ }^{1}$, S. Pouchla ${ }^{2}$, H. Vinohradska ${ }^{3}$, P. Konecna ${ }^{1}$, Z. Dolezel ${ }^{1}$

${ }^{1}$ Department of Pediatrics, University Hospital Brno and Medical Faculty of Masaryk University, ${ }^{2}$ Center of Molecular Biology and Gene Therapy,

${ }^{3}$ Department of Biochemistry, University Hospital Brno, Brno, Czech Republic

Background and aims: Phenylketonuria (PKU;OMIM261600)isaninheritedmetabolicdisease due to a deficiecy of hepatic phenylalaninhydroxylase $(\mathrm{PAH} ; 12 \mathrm{q} 24.1)$. Phenylalanine accumulation can lead to cognitive impairment. Tetrahydrobiopterin $(\mathrm{BH} 4)$ responsiveness in patients with specific mutations in the $\mathrm{PAH}$ gene is a subtype of hyperfenylalaninaemia (HPA) charecterised by a positive $\mathrm{BH} 4$ loading test.

Methods: We tested 21 patients with HPA/PKU, 4-39 years of age, selected (based on genotype) as a potentially $\mathrm{BH} 4$ responsive and loaded with $\mathrm{BH} 4$ (20mg/kg).

Results: Overall 9/21 showed positive response of more than $30 \%$ decrease in blood Phe levels $8 \mathrm{~h}$ after $\mathrm{BH} 4$ challenge, and $7 / 21$ showed this decrease after $24 \mathrm{~h}$. The majority of the responsive patients belong to mild HPA (11/16). Genotype analysis confirms the involvement in the response of specific mutations. In p. E390G, p.A300S, p. A403V, p.Y414C, p.I306V, p.G272X, p.I65T, p.R261Q and p.Y387H mutations were $100 \%$ associated with $\mathrm{BH} 4$ responsiveness. The p.R158Q mutation was inconstantly responsive. The EX5del-4kb mutation was responsive regardless of the second allele (p.A403V and p.R408W). In patient with mild PKU and novel mutation p.K396R (genotyp p.R408W/p. K396R) no responsiveness was noticed.

Conclusions: According to recent estimates, a group of individuals may benefit from $\mathrm{BH} 4$ substitution, eliminating the need of life-long dietary restrictios. The best responders were patients with mild HPA. 